\title{
Graded changes in evoked response (P300) amplitude as a function of cognitive activity*
}

\author{
E. DONCHIN, M. KUBOVY, M. KUTAS, R. JOHNSON, JR., and R. I. HERNING \\ University of Ilinois at Urbana-Champaign, Champaign, Ilinois 61820
}

\begin{abstract}
Using a stepwise discriminant analysis, we demonstrate that the amplitude of $P 300$ is a graded function of the complexity of information processing required of a $S$ following a stimulus. This relationship between cognitive complexity and P300 is apparent only when the $S$ is not pressed to generate fast and accurate discriminant responses to the stimuli. Under a reaction-time regime, a large P300 is elicited independently of stimulus predictability or cognitive complexity. It seems that P300 is a measure of the amount of activity of a general-purpose cortical processor.
\end{abstract}

Early investigators of the human average evoked potential (AEP) (Katzman, 1964) tended to consider these potentials as reflecting "sensory" cortical activity triggered by the input volleys associated with a stimulus. When complex psychological processes, such as "attention," were shown to affect the shape of the AEP, it was assumed that these effects were due to the modulation of cortical excitability (Haider et al, 1964; Donchin \& Lindsley, 1966). Since 1965, however, much evidence has accumulated that certain components of the human AEP are associated with intracortical data processing rather than with the registration of stimuli. One such component, a positive-going complex, with a latency to the peak of approximately $300 \mathrm{msec}$, is often labeled P300. The amplitude of P300 can be enhanced or diminished by the manipulation of the S's task or by the circumstances in which the stimulus is presented, although it is little affected by the physical properties of the stimulus (Sutton et al, 1965; Sutton et al, 1967; Hillyard et al, 1971; Harter \& Salmon, 1972; Ritter \& Vaughan, 1969). Visual and auditory stimuli can be interchanged in a task without appreciable effect on P300 (Donchin \& Cohen, 1967; Donchin, 1968; Donchin \& Smith, 1970; Smith et al, 1970). It has been shown that the early sensory components of the AEP can be dissociated from P300 (Rohrbaugh et al, 1972; Rohrbaugh, 1973). Furthermore, several investigators have reported that P300 can be elicited in the absence of a stimulus (Sutton et al, 1967; Klinke et al, 1968; Weinberg et al, 1970; Picton et al, in press).

While it seems clear that P300 reflects endogenous cortical processes, there is little consensus concerning their nature. There are those who suggest that P300 reflects undifferentiated arousal (Karlin, 1970; Näätänen, 1969a, b); this position, however is not

*Supported by Grant OEG-5-70-0030 (508) from the U.S. Office of Education and by Grant NSF-GB-30999 from the National Science Foundation, both to E. Donchin, as well as by the University of Illinois Research Board. W. R. Mead and Charles Warren participated in early phases of this project. We appreciate the help of Esther Williams and Sara Bruce of the Psychology Department's computing facility. Preliminary reports on this investigation were read at the meetings of the Psychonomic Society, in St. Louis, Mo., in November 1971 and November 1972. consistent with several recent studies (Donchin \& Cohen, 1969; Donchin et al, 1972; Harter \& Salmon, 1972; see also Tueting \& Sutton, 1971). Others have proposed that P300 represents the output signal of a cortical processor. Thus, several investigators have related $P 300$ to the activity of a "mismatch" or a "novelty" detector (e.g., Ritter \& Vaughan, 1969; Hillyard et al, 1971). It has been variously suggested that $\mathbf{P 3 0 0}$ is associated with the "resolution of uncertainty" or with "information delivery" (Sutton et al, 1965; Donchin \& Cohen, 1967). An alternative view is that $\mathbf{P} 300$ reflects in a more general way the amount of activity of a processor of broad capabilities, which is invoked by any decision or cognitive act of the S (Smith et al, 1970). The results reported here are consistent with the latter view.

In part, the problem in characterizing the psychological processes associated with $\mathrm{P300}$ is that most previous experiments were dichotomous: the Es demonstrated either the presence or absence of P300, under diverse experimental conditions. Very few investigators have developed experimental procedures where P300 amplitude can be plotted as a function of some independent variable (Hillyard et al, 1971; Paul \& Sutton, 1972; Ritter et al, in press). It is such an attempt that is described in the present report.

We based our procedure on the prediction task used by Sutton and his co-workers (1965). Sutton's data were clear: stimuli embedded in an unpredictable series elicit a P300 larger than that elicited by physically identical stimuli in a predictable series. We modified Sutton's procedure by varying the degree to which the $S$ could utilize information from preceding trials in predicting the next trial's outcome.

\section{METHOD}

A trial began with a 100 -msec warning tone, followed after $1,400 \mathrm{msec}$ by a 100 -msec flash which illuminated either the letter "A" or the letter "B." On "guess" sessions, Ss were required to predict, prior to the warning tone, which of the letters would follow. On "reaction time" sessions, the Ss pressed one of two buttons, with the left or right thumb, depending on the letter presented. A force of $450 \mathrm{~g}$ was required to close each of the two microswitches. On these sessions both speed and correctness of response were heavily stressed.

Trials were presented in one of four sequence types. In the 


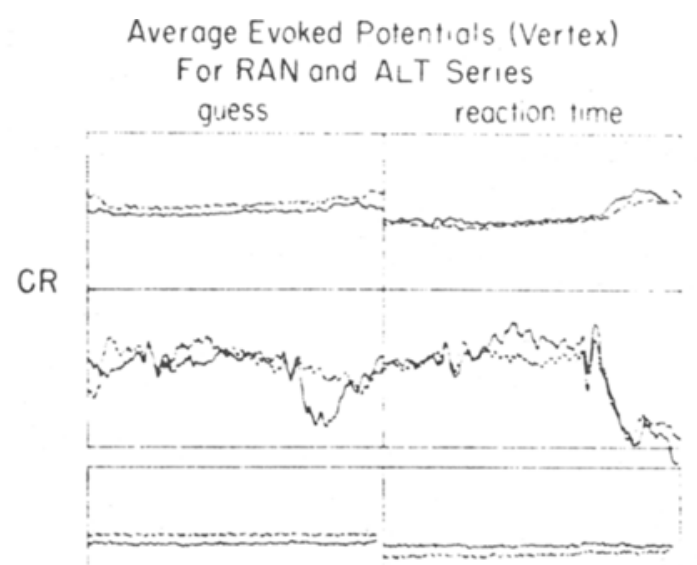

$D E \ldots$

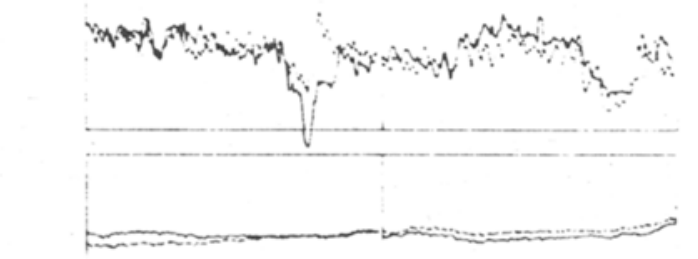

$B E$

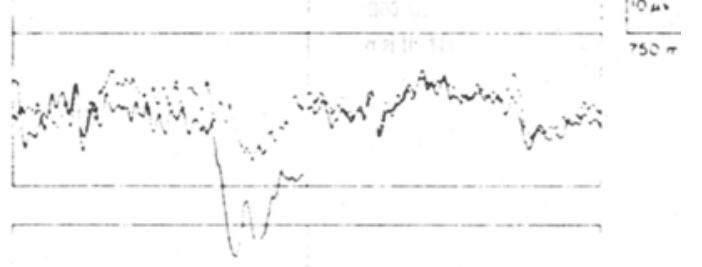

OS
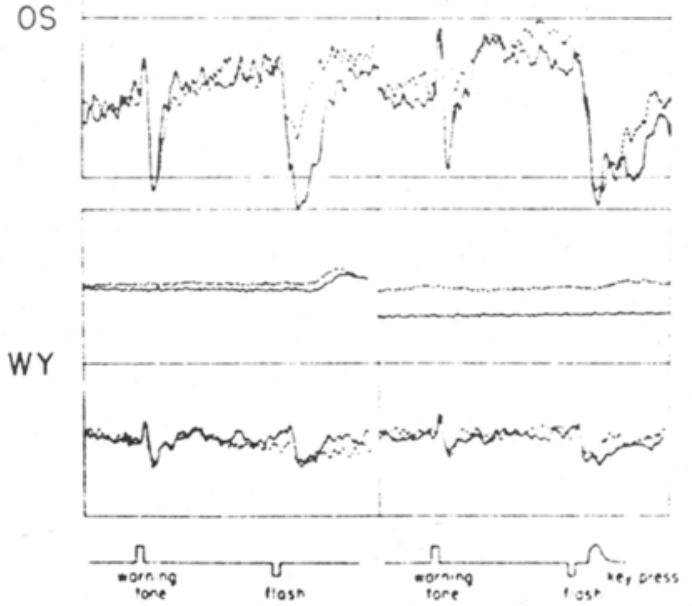

Fig. 1. Average evoked potentials recorded from vertex. Each pair of curves recorded from one $S$. The solid line represents a RAN series, the dotted line represents an ALT series. Number of trials in each AEP $=50$. Negativity upwards curves were superimposed by aligning the evoked responses to the warning stimulus. Note amplitude difference at $P 300$, in the range of $200-400 \mathrm{msec}$ after the flash in the guess conditions.

alternating sequence, the $A s$ and $B s$ simply alternated on successive trials (e.g., $A, B, A, B, A, B, A, B, \ldots$ etc.). The random sequence was a sequence of independent Bernoulli trials, the probability of an $A$ being equal to the probability of a $B\left(P_{a}=\right.$ $\left.\mathbf{P}_{b}=.50\right)$. There were also series in which the $A s$ and $B s$ were presented in a fixed pattern, usually a nine-element-long sequence which was cyclically repeated (e.g.; $B, B, A, B, A, B, A, A, B$ and again $B, B, A, B \ldots$ etc.). Finally, there was a pseudopattern sequence, generated by using the nine-item patterns and cumulatively changing a randomly selected element on each cycle

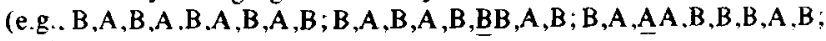
etc.).

We varied systematically the sequence of As and Bs that were presented to the $\mathrm{S}$. Four different sequence types were presented in five different experimental series as follows: (a) Alternating series (ALT)--an alternating sequence was used, and the $S$ was so informed. (b) Known pattern (LRN)-a fixed pattern of As and $B s$ was used. The $S$ was handed a sheet of paper with the correct pattern and was asked to master it before the series began. (c) Unknown pattern (PAT) - a fixed pattern sequence was used, as in the LRN series. However, the $S$ looked for, and usually discovered, the pattern during the series. (d) Search series (SHF) - a pseudopattern sequence was used. The $S$ was given the same instructions he was given during the PAT series; that is, he looked for. and could not find, a pattern during the series. (e) Random series (RAN)-a random sequence was used, and the $S$ was so informed. Before engaging in either (c) or (d), the $S$ was informed that the pattern might be too difficult and that he might fail to find it. All series were 50 trials long (except the PAT series, which was 100 trials long).

Ten University of Illinois undergraduates (six of them male) served as Ss. Thirteen Beckman biopotential skin electrodes (No. 6509), filled with Beckman electrode paste, were affixed to the S's head. This paper reports only the results recorded at the vertex $\left(C_{z}\right)$ referred to a pair of linked electrodes placed on the mastoid processes. The $\mathrm{S}$ was grounded by means of midforehead electrode. The electrooculogram (EOG) was recorded between a supraorbital and a canthal electrode. Electrode impedances did not exceed $10 \mathrm{k}$ ohms. The EEG was amplified with Brush amplifiers (No. 13-4218-00), set to a bandpass of $.1-30 \mathrm{~Hz}$. Data were recorded on an HP3955 FM tape recorder and averaged with an IBM 1800 computer.

The $\mathrm{S}$ was seated in a comfortable reclining lounge chair in a Faraday cage located in a darkened room. He looked into an Iconix three-field look-into exposure box (No.6134) with cold cathode fluorescent lamps (No. 6109-3). A blank fixation field subtending $3 \mathrm{deg} 44 \mathrm{~min}$ was on for the duration of the experiment (luminance: $10.8 \mathrm{~mL}$ ). The two other fields (subtending of the same visual angle) contained the " $A$ " and the " $\mathrm{B}$," the black-on-white letters subtended $55 \mathrm{~min}$ horizontally and $1 \mathrm{deg} 26 \mathrm{~min}$ vertically. The luminance of the fields was $17.2 \mathrm{~mL}$. A $1-\mathrm{kHz}$ tone, generated by a Hewlett-Packard pushbutton oscillator (No. 214A), was amplified and drove a speaker producing an SPL of $68 \mathrm{~dB}$ re $.0002 \mu \mathrm{bar}$.

In the reaction time sessions, the letter reappeared if the $S$ did not respond within a critical interval $(250$ to $320 \mathrm{msec}$ for different $S s)$. The critical interval for each $S$ was selected during the training session. Trials were initiated irregularly (mean intertrial interval, $5.8 \mathrm{sec}$; standard deviation, $.7 \mathrm{sec}$; range, $5.0-7.2 \mathrm{sec}$ ). The schedules were controlled by an Iconix logic system.

Each $S$ was run in three different (2-h) sessions: a training session, a "reaction time" session in which the $S$ was instructed to respond as fast as he could, and a "guess" session.

\section{RESULTS}

Figure 1 presents the AEPs obtained from five of our Ss in the reaction time and the guess sessions for the RAN and ALT series (similar data were obtained from the other five Ss). In each case, the two superimposed traces represent the vertex AEPs (solid lines for RAN, dotted lines for ALT), the corresponding EOG is 
displayed above the vertex data. As has been previously reported by Sutton and his co-workers (1965), P300 is considerably larger when the stimuli are presented in a RAN sequence.

This difference in P300 is greatly diminished when a reaction time demand is imposed on the $\mathrm{S}$. A large $\mathrm{P} 300$ is elicited during the ALT series if the S must respond speedily and accurately to the imperative stimulus. In view of these data, it may be appropriate to reject the notion that there is a necessary relationship between the "uncertainty" the $S$ has concerning the stimulus and P300 amplitude. There is, of course, no "uncertainty" in the ALT series during the reaction time experiment, and yet these highly predictable stimuli elicit a large P300. Although uncertainty may be sufficient to enhance P300, it is clearly not a necessary condition for its elicitation.

Since P300 was different in the two extreme conditions of the guess sessions, RAN and ALT, we investigated the relative amplitudes of $P 300$ in the other three conditions. Visual inspection of the guess data revealed in all Ss that $\mathbf{P} 300$ amplitude increases from a minimum at the ALT series to a maximum for the RAN series through LRN, PAT, and SHF, in this order.

To circumvent the problem of visually locating the $\mathrm{P} 300$, we resorted to the use of the stepwise discriminant analysis (SWDA) procedure (Dixon, 1970; Tatsuoka, 1971). For each experimental trial, the successive measures of voltage between two electrodes obtained at equally spaced time points following the initial eliciting event constitute a vector $\bar{X}$, with elements $x_{i j k}$, where $i=1, N(N$ being the number of time points in a given evoked response), $j=1, P$ (where $P$ is the number of times the stimulus has been presented), and $\mathrm{k}=1, \mathrm{~S}$ (where $\mathrm{S}$ is the number of experimental conditions). In our experiment, $S=5$ (the number of the experimental series), $P=50$ (except in the PAT series, where $P=100$ ), and $N=75$. These 75 points spanned a $2,700-\mathrm{msec}$ interval. The values of the $\mathrm{N}$ points in time at which the EEG was sampled will be called "variables."

The SWDA program is assigned the following task: given a set of $S$ experimental conditions, to select an M-element subset of the $N$ variables so that a linear combination of these $\mathbf{M}$ variables (the discriminant function) will maximize the between-condition variance of that combination relative to the within-condition variance over the $P$ trials. The value of the discriminant function is, in a sense, a measure of the similarity of any vector to the mean vectors (the AEPs) representing the experimental conditions. In other words, the task is to develop a classification rule which could be applied to newly obtained vectors whose experimental condition of origin is unknown. In terms of the present experiment, we were asking the program to consider the 50 vectors associated, respectively, with the RAN and the ALT series and to develop a classification rule which could be applied to each of the vectors associated with the other three experimental conditions. We are, in effect, trying to assess the extent to which a vector, obtained, say, during the LRN sequence, is "like" the vectors obtained during the RAN series.

The appropriateness of SWDA for the analysis of evoked potential data is supported by other work: Donchin and his coworkers have successfully tested it on simulated data ${ }^{2}$ and have found it useful in their analyses of earlier experiments (Donchin, 1969a, b; Donchin et al, 1970).

For each of our $10 \mathrm{Ss}$, we obtained such a discriminant function, discriminating between RAN and ALT. We allowed a maximum of six variables (time points) for each function. As can be expected from Fig. 1, in most cases the first variables to be selected for use in this discrimination were from the P300 region. The value of this function for each of the individual trial vectors was computed, as was the discriminant function at the group AEPs. The resulting values were used to compute the probability that the trial vector was drawn from the RAN condition or the ALT condition. We could thus determine the proportion of trials in each of the experimental conditions classified as coming from the RAN population. For each $S$, the majority of the RAN trials were classified as RAN, the majority of ALT trials were not classified as RAN. Moreover, the other three conditions were ranked in terms of the proportion of RAN-like vectors they generated. It should be emphasized that there is no circularity in this argument. The classified vectors associated with LRN, SHF, and PAT trials were not used in developing the RAN/ALT classification: they were "new" data as far as this classification was concerned. Interestingly, most LRN trials were classified as ALT, most SHF trials were classified RAN, and the PAT data were often evenly divided among the ALT and RAN groups.

In Fig. 2 we plot the value of the discriminant function evaluated for the AEP of each of the experimental series as a function of the experimental conditions. Strikingly, for all $10 \mathrm{Ss}$, the AEPs are ordered RAN to ALT through LRN, PAT, and SHF. This method provides a ready way for presenting control data. Consider, for example, the panel labeled EOG in Fig. 2. In it, we plot the values of the vertex discriminant function applied to the corresponding EOG data. It is clear that the systematic relations observed in the vertex cannot be accounted for as EOG artifacts. Finally, in the panel labeled "reaction time" in Fig. 2, we plot the values of the discriminant function applied to the RAN and ALT AEPs obtained during reaction time sessions. As can be expected from the data presented in Fig. 1, the RAN and ALT AEPs are indiscriminable.

\section{DISCUSSION}

It is evident, then, that the vertex P300 somehow orders the five experimental conditions in the guess sessions. Thus, a dimension can be defined with RAN and ALT as its extremes, the other three conditions 
"Guess" Descriminont Function Evaluated or Vorious AEPS

Vertex

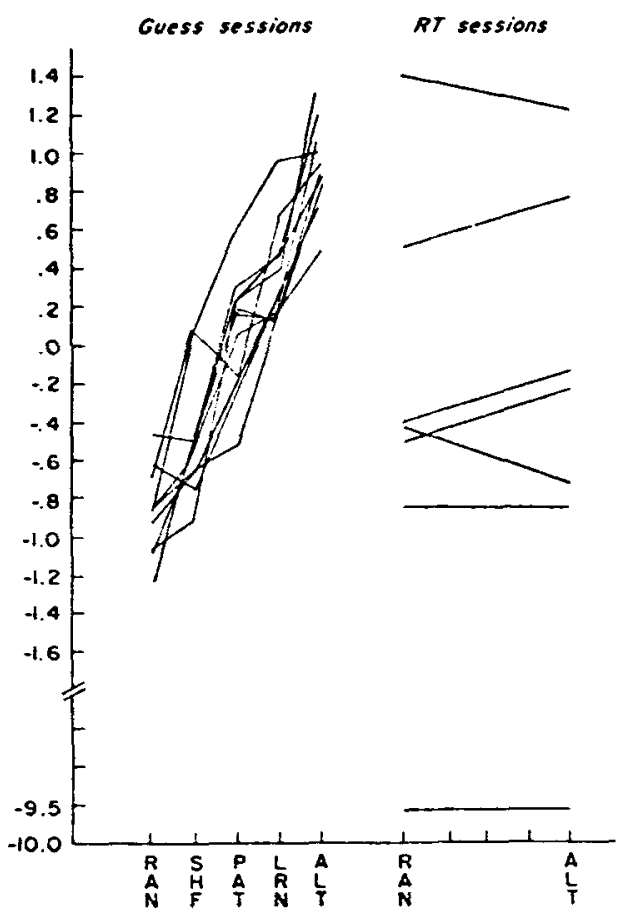

EOG

Guess sessions

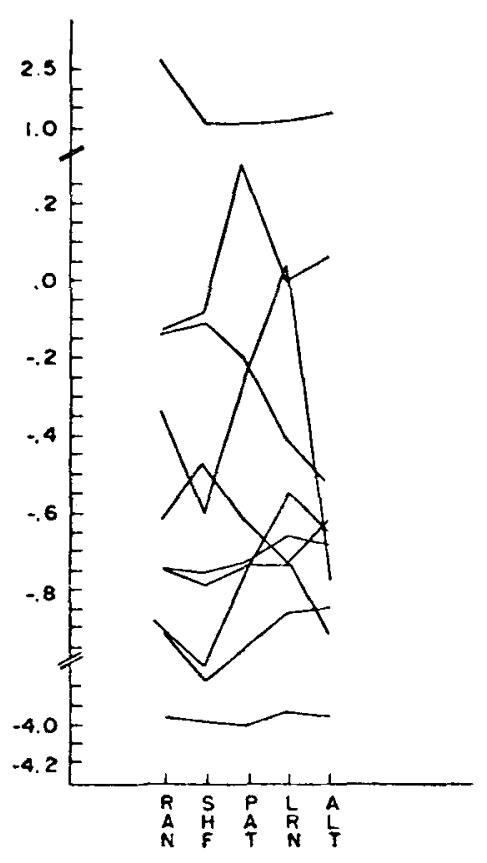

Fig. 2. Values of the discriminant function developed on the basis of the RAN and ALT guess series evaluated at different AEPs. Each line represents the data from one $S$ for one class of experimental conditions. Data are shown for 10 Ss for the guess sessions for both the vertex and the EOG AEPs and for $7 \mathrm{Ss}$ for the reaction time sessions. Note how value of discriminant function is ordered for the five series for the guess sessions vertex data and for all 10 Ss. This ordering does not appear at the EOG. Also note that the function discriminating RAN from ALT during a guess session fails to discriminate RAN and ALT during a reaction time session. falling on intermediate points. The effects of the experimental manipulations are cancelled when the $S$ is required to respond to the stimuli. What is the nature of the variable which controls this dimension? One possibility is suggested by Squires et al's (1973) report that stimuli providing "disconfirming feedback" elicit a large P300. When our Ss predict an " $A$ " and receive a " $B$," their prediction is, of course, disconfirmed. Indeed, the number of errors made per series correlates well with P300 amplitude. There are virtually no errors in the ALT and LRN series, few errors in the PAT series (mostly in its early part), and there are equal numbers of errors in the SHF and RAN series (approximately 50\%). Visual inspection of "correct" vs "incorrect" AEPs for several experimental conditions revealed no systematic effects of that variable. Furthermore, when the SWDA was applied specifically to the "correct" trial data alone, disregarding incorrect trials, the results were the same as those reported above for the unselected AEPs. It is possible that in our experiment, where the $S$ needed information on the sequence with which stimuli were presented, both errors and correct predictions were equally useful and therefore both "confirming" and "disconfirming" stimuli were associated with large P300.

One needs, it seems, to consider the specifics of the S's task if the amplitude of the elicited P300 is to be understood. Consider our $S$ s to be processing the information provided on each trial in order to predict the next stimulus. Each of the five conditions requires a different amount of processing. Thus, for example, in the ALT series, a mere registration of the stimulus was sufficient for the next prediction. In the LRN series, in which no errors were committed, the successful prediction may require, in addition, a search of the S's memory to determine the present position in the sequence. Similar considerations apply to the PAT and SHF series. It is, perhaps, difficult to understand what processing is conducted by the $\mathrm{S}$ during the RAN series. However, considerable evidence exists that, when faced with random sequences, $\mathrm{Ss}$ ' prediction behavior is guided by the assumption that there are short-term regularities in a random sequence (see Tversky \& Kahneman, 1971).

We can give only post hoc explanations for the cancelling of the effects of the sequence variables on P300 by the imposition of a reaction time demand on the $S$. The S's task is, of course, different. He must decide when to respond, and to be careful to respond fast and accurately, and these task demands lead to an enhancement of P300, independently of stimulus predictability. Yet, whatever the explanation, this result is important in that it shows that "unpredictability" is not a necessary condition for the enhancement of $\mathrm{P} 300$.

In view of the diversity of the P300 function that our data reveal, it seems that a reasonable approximation is to assume that $\mathrm{P} 300$ reflects the activity of a general-purpose processor which is invoked on demand by a host of data processing requirements. The processor might be conceived of as analogous to a floating point processor (FPP) attached to a computer. The FPP may be invoked by many different programs whenever they 
require computations of a certain accuracy. Nothing else needs to be common to the invoking programs. If we have a utilization meter on the FPP, we shall find its utilization a function of the amount of floating point processing required by various tasks imposed on the computer. We are suggesting that it is conceivable that in P300 we have such a utilization measure for an intracortical processor. In other accounts of P300, there is, it seems, the implication that P300 is the output of an intracortical processor. Consider, for example, Squires et al's (1973) statement that, "The behavior of P3(00) ... seems compatible with a model ... which specifies that P3(00) varies directly with the closeness of the template match and inversely with the degree of expectancy ...." They go on to discuss P300 as the sum of the "output" of various processing stages, the amplitude of which, in their model, determines the S's decision. We do not share this view.

The analogy to a utilization measure for an intracortical processor suggests the nature of the research required if we are to understand the functional significance of $\mathrm{P} 300$. If we assume that we have a measure of Processor X's utilization by an information-processing system, $A$, then, even though we know nothing further about Processor $X$, we might be able to identify its function if we present System A with a series of tasks which, in a controlled fashion, call for different types of processing. Systematic analysis of graded amplitude changes in P300 such as we report should reveal how the utilization of Processor $X$ is a function of specific processing tasks. One is led, therefore, to study such variables as the complexity of memory search, or the effects of organizational complexity of serial patterns on P300. We assume that one, or several, of these variables will prove determinative of $\mathbf{P} 300$ amplitude.

\section{REFERENCES}

Dixon, W. J. (Ed.) BMD-biomedical computer programs. Los Angeles: University of California Press, 1970.

Donchin, E. Average evoked potentials and uncertainty resolution. Psychonomic Science, 1968, 12, 103.

Donchin, E. Data analysis techniques in average evoked potential research. In E. Donchin and D. B. Lindsley (Eds.), Average evoked potentials: Methods, results, and evaluations. Washington, D.C: NASA, SP-191, 1969a. Pp. 199-236.

Donchin, E. Discriminant analysis in average evoked response studies: The study of single trial data. Electroencephalography \& Clinical Neurophysiology, 1969b, 27, 311-314.

Donchin, E. Methodological issues in CNV research. Electroencephalography \& Clinical Neurophysiology, 1973, Suppl. 33, in press.

Donchin, E., Callaway, E., \& Jones, R. T. Auditory evoked potential variability in schizophrenia, II. The application of discriminant analysis. Electroencephalography \& Clinical Neurophysiology, 1970, 29, 429.440.

Donchin, E., \& Cohen, L. Averaged evoked potentials and intramodality selective attention. Electroencephalography \& Clinical Neurophysiology, 1967, 22, 537-546.

Donchin, E., \& Cohen, L. Further reply to Näätänen. Perceptual \& Motor Skills, 1969, 29, 270.
Donchin, E., Gerbrandt, L., Leifer, L., \& Tucker, L. Is the contingent negative variation contingent on a motor response? Psychophysiology, 1972, 9, 178-188.

Donchin, E., \& Lindsley, D. B. Average evoked potentials and reaction times to visual stimuli. Electroencephalography \& Clinical Neurophysiology, 1966, 20, 217-223.

Donchin, E., \& Smith, D. B. D. The contingent negative variation and the late positive wave of the average evoked potentials. Electroencephalography \& Neurophysiology, 1970, 29, 201-203.

Haider, M., Spong, P., \& Lindsley, D. B. Attention, vigilance, and cortical evoked potentials in humans. Science, 1964, 145, 180-182.

Harter, M. R., \& Salmon, L. E. Intramodality selective attention and evoked cortical potentials to randomly presented patterns. Electroencephalography \& Clinical Neurophysiology, $1972,32,605-613$.

Hillyard, S., Squires, C., Bauer, J., \& Lindsay, P. Evoked potential correlates of auditory signal detection. Science, $1971,172,1357-1360$.

Karlin, L. Cognition, preparation, and sensory-evoked potentials. Psychological Bulletin, 1970, 73, 122-136.

Katzman, R. (Ed.) Sensory evoked response in man. Annals of the New York Academy of Sciences, 1964, 112, 546.

Klinke, R., Fruhstorfer, H., \& Finkenzeller, P. Evoked responses as a function of external and stored information. Electroencephalography \& Clinical Neurophysiology, 1968, $25,119-122$.

Näätänen, R. Anticipation of relevant stimuli and evoked potentials: A comment on Donchin's and Cohen's "average evoked potentials and intramodality selective attention." Perceptual \& Motor Skills, 1969a, 28, 639-646.

Näätänen, R. Anticipation of relevant stimuli and evoked potentials: A reply to Donchin and Cohen. Perceptual \& Motor Skills, 1969b, 29, 233-234.

Paul, D. D., \& Sutton, S. Evoked potential correlates of response criterion in auditory signal detection. Science, 1972, 177, 362.

Picton, T. W., Hillyard, S. A., \& Galambos, R. Cortical evoked responses to omitted stimuli. In M. N. Livanov (Ed.), Major problems of brain electrophysiology. USSR Academy of Sciences, in press.

Ritter, W., Simson, R., Vaughan, H. G., Jr. Association cortex potentials and reaction time in auditory discrimination. Electroencephalography \& Clinical Neurophysiology, in press.

Ritter, W., \& Vaughan, H. G. Average evoked responses in vigilance and discrimination: A reassessment. Science, 1969, $164,326-328$.

Rohrbaugh, J. Cortical potentials associated with decision-making. Unpublished doctoral dissertation, University of Illinois, 1972.

Rohrbaugh, J., Donchin, E., \& Eriksen, C. W. On the differential effect of cognitive variables on early or late components of visual evoked response. Paper presented at the meeting of the EEG Society, Houston, October 1972.

Smith, D. B. D., Donchin, E., Cohen, L., \& Starr, A. Auditory evoked potentials in man during selective binaural listening. Electroencephalography \& Clinical Neurophysiology, 1970, $28,146 \cdot 152$.

Squires, K. C., Hillyard, S. A., \& Lindsay, P. H. Cortical potentials evoked by confirming and disconfirming feedback following an auditory discrimination. Perception \& Psychophysics, 1973, 13, 25-31.

Sutton, S., Braren, N., Zubin, J., \& John, E. R. Evoked-potential correlates of stimulus uncertainty. Science, 1965, 150, 1187-1188.

Sutton, S., Tueting, P., Zubin, J., \& John, E. R. Information delivery and the sensory evoked potential. Science, 1967, 155, 1436-1439

Tatsuoka, M. M. Multivariate analysis. New York: Wiley, 1971. Tueting, P., \& Sutton, S. The relationship between pre-stimulus 
negative shifts and the late components of the average evoked potential. Paper presented at the 4th International Symposium on Attention and Performance, Boulder. Colorado, 1971.

Tueting, P., Sutton, S., \& Zubin, J. Quantitative evoked potential correlates of the probability of events. Psychophysjology, 1970, 7, 385-394.

Tversky, A., \& Kahneman, D. Subjective probability: A judgment of representativeness. ORI Research Bulletin, 1971, 11 , No. 2.

Weinberg, H.. Waiter, W. G., \& Crow, J. H. Intracerebral events in humans related to imaginary stimuli. Electroencephalography \& Clinical Neurophysiology, 1970, $29,1-9$.

\section{NOTES}

1. As the experimental paradigm included the usage of a warning stimulus and a "foreperiod," we provided for an analysis of CNVs as well as for P300. We shall report elsewhere results concerning the CNV. Here we can only say that these were far more variable and far less orderly than our P300 data. For a discussion of preliminary analyses of CNVs in a similar experiment, see Donchin (in press).

2. Donchin, E, \& Herning, R. I. A simulated study of the efficacy of stepwise discriminant analysis of evoked response data. In preparation.

(Received for publication April 13, 1973; revision received April 30, 1973.) 